\title{
Voluntary action alters the perception of visual illusions
}

\author{
Matti Vuorre $^{1}$ (D) Janet Metcalfe ${ }^{1}$
}

Published online: 27 April 2017

(C) The Psychonomic Society, Inc. 2017

\begin{abstract}
Intentional binding" refers to the finding that people judge voluntary actions and their effects as having occurred closer together in time than two passively observed events. If this effect reflects subjectively compressed time, then timedependent visual illusions should be altered by voluntary initiation. To test this hypothesis, we showed participants displays that result in particular motion illusions when presented at short interstimulus intervals (ISIs). In Experiment 1 we used apparent motion, which is perceived only at very short ISIs; Experiments $2 \mathrm{a}$ and $2 \mathrm{~b}$ used the Ternus display, which results in different motion illusions depending on the ISI. In support of the time compression hypothesis, when they voluntarily initiated the displays, people persisted in seeing the motion illusions associated with short ISIs at longer ISIs than had been the case during passive viewing. A control experiment indicated that this effect was not due to predictability or increased attention. Instead, voluntary action altered motion illusions, despite their purported cognitive impenetrability.
\end{abstract}

Keywords Time perception · Visual perception · Agency · Volition · Action

When people judge the timing of their voluntary action and its subsequent effect—say, a button press causing a beep — they

The original version of this article was revised: Figures 2-6 in the published article were not the ones submitted by the authors. Specifically, they were of lower quality than the submitted images, and missed credible intervals from each panel A.

Matti Vuorre

mv2521@ columbia.edu

1 Psychology Department, Columbia University, 365 Schermerhorn, 1190 Amsterdam Avenue, New York, NY 10027, USA retrospectively judge the action and beep as having occurred closer together in time than if they had passively observed similar events: Voluntary action seems to compress time. We sought to determine whether this effect (called "intentional binding"; Haggard, Clark, \& Kalogeras, 2002; Moore \& Obhi, 2012) occurs because time is perceptually compressed by voluntary actions, or whether it only seems so when judged retrospectively.

Although the idea that subjective time can be compressed might seem counterintuitive, people often report the opposite - time slowing (i.e., expanding) — during life-threatening experiences. To investigate whether time really expanded during such events, Stetson, Fiesta, and Eagleman (2007) had participants jump off a high tower into a net far below, a manipulation that reliably induced retrospective reports of expanded time. They interposed a visual task during the fall in which digits were presented very quickly - so quickly that under normal conditions the digits fuse and become unreadable. If time had really expanded, participants should have been able to read the digits. However, people's perceptions of the digits were unchanged. The digits still fused, indicating that the feeling of expanded time during this frightening experience was retrospective rather than real.

Voluntary actions are thought to compress time, rather than expand it (Haggard et al., 2002). The evidence for time compression, though, is almost exclusively retrospective, and dependent upon reports of when the events occurred - using the Libet clock methodology to elicit retrospective reports of when actions and their effects occurred (Haggard et al., 2002) - or of the time interval between two events (e.g., Engbert, Wohlschläger, Thomas, \& Haggard, 2007, who asked participants to verbally estimate the action-effect interval on each trial).

In the only study (Wenke \& Haggard, 2009) suggesting that the effect might not be entirely retrospective, participants received two closely spaced successive electric shocks on the 
finger that moved in the voluntary action condition. In this case, participants needed a longer interstimulus interval (ISI) between the shocks to identify them as nonsimultaneous following voluntary action. This result was interpreted by the authors as support for the temporal compression hypothesis. But while it is consistent with the temporal compression hypothesis, this result could also have occurred because impaired discrimination occurred for reasons that had nothing to do with time. There may instead have been sensory overload or numbing of the finger, insofar as the shocks were delivered on the finger that was both the source of the action and the locus of the temporal discrimination (e.g., Williams \& Chapman, 2002). Although these perceptual effects suggest that time compression might occur as a result of voluntary action, the inference would be more convincing if a phenomenal or perceptual change were to be manifested in a sensory modality and bodily location that were removed from the action itself.

We conjectured that if time were subjectively compressed by voluntary action, as Wenke and Haggard's (2009) study suggests, then people might perceive time-dependent illusory visual motion differently when they voluntarily initiated the events, as compared to when they passively viewed the same events. Specifically, the perceptual time compression hypothesis predicts that people would observe illusions associated with short ISIs at longer objective ISIs following voluntary action than when they passively viewed the same stimuli.

In Experiment 1, participants observed two successive, spatially offset circles, which at very short ISIs result in perceived apparent motion (e.g., Kolers \& Pomerantz, 1971; Wertheimer, 1912; see Fig. 1A). We varied the ISI over a range in which at the short end people see apparent motion, and at the long end see two separate stationary circles. After each trial, we asked people to indicate whether or not they had observed apparent motion. We varied whether they initiated the display with a voluntary button press or watched the display passively. To replicate previous studies of intentional binding using time estimation reports, in a separate task, using the same stimuli, participants retrospectively estimated the ISI. If voluntary action has the effect of subjective time compression, both the retrospective reports and the illusory perceptual-motion effects, just outlined, would be in evidence: Voluntary action would result in perceived apparent motion at longer ISIs, as well as in longer overall estimates of the ISIs, than would passive viewing. If intentional binding were only retrospective, however, there should be no difference in the perceptual reports of illusory motion, but there should still be a difference in participants' retrospective reports of time as a function of whether or not they had initiated the movement.

In Experiment 2a we used the Ternus illusion, in which two horizontally aligned circles are presented such that the rightmost circle is shown mid-screen (Ternus, 1926). After an ISI, the two circles are shown again, such that the left circle is mid- screen and the rightmost circle is offset to the right (Fig. 1B). With sufficiently short ISIs, observers perceive the leftmost circle as leapfrogging over the center circle to land on its other side ("element motion"). At longer ISIs, the two circles appear to move in tandem ("group motion"). We varied both the ISI and whether participants voluntarily initiated the display or just passively watched, to discover whether they would observe element or group motion. To ensure the reliability of our findings, we then conducted a direct replication of Experiment 2a (Exp. 2b).

\section{Experiment 1}

\section{Method}

Participants Twenty-four Columbia University undergraduates participated for course credit. We aimed for a sample size similar to those reported in previous studies (Engbert et al., 2007, $n=18$; Wenke \& Haggard, 2009, $n=19$ ). One participant quit the study before the interval estimation task, but otherwise all participants completed both tasks, and they always completed the apparent-motion task first. We chose this order to ensure that participants would not carry perceptual learning or response biases from the interval estimation task to the apparent-motion task, which was the main target of inference in the present study.

The experiment was approved by the Columbia University Internal Review Board and was carried out in accordance with the Psychonomic Society ethical guidelines and with the Declaration of Helsinki.

Apparent-motion task The experiment was based on a 2 (Action Condition: voluntary action, no action) $\times 8$ (ISI: 33 , $50,83,100,133,150,200,300 \mathrm{~ms}$ ) within-participants factorial design. The primary dependent measure - judgment of apparent motion - was a binary (yes or no) response. Action condition was manipulated between four counterbalanced blocks of 80 trials each. ISI was randomized between trials, resulting in 20 trials of each ISI-action condition pair, and a total of 320 trials per participant.

Participants completed the experiment individually, on an Apple iMac computer running at a $60-\mathrm{Hz}$ refresh rate, in a dark testing room. They were seated approximately $60 \mathrm{~cm}$ from the monitor and wore headphones throughout the study. At the beginning of the study, the participants read through the experiment instructions and were instructed that the experiment was about motion perception. Before each block, onscreen instructions indicated which action condition the next block would represent. For voluntary-action blocks, the instructions read: "In the next trials, press the mouse button to initiate the display. Press the mouse button whenever you wish to do so." For no-action blocks, the instructions read: "For the 


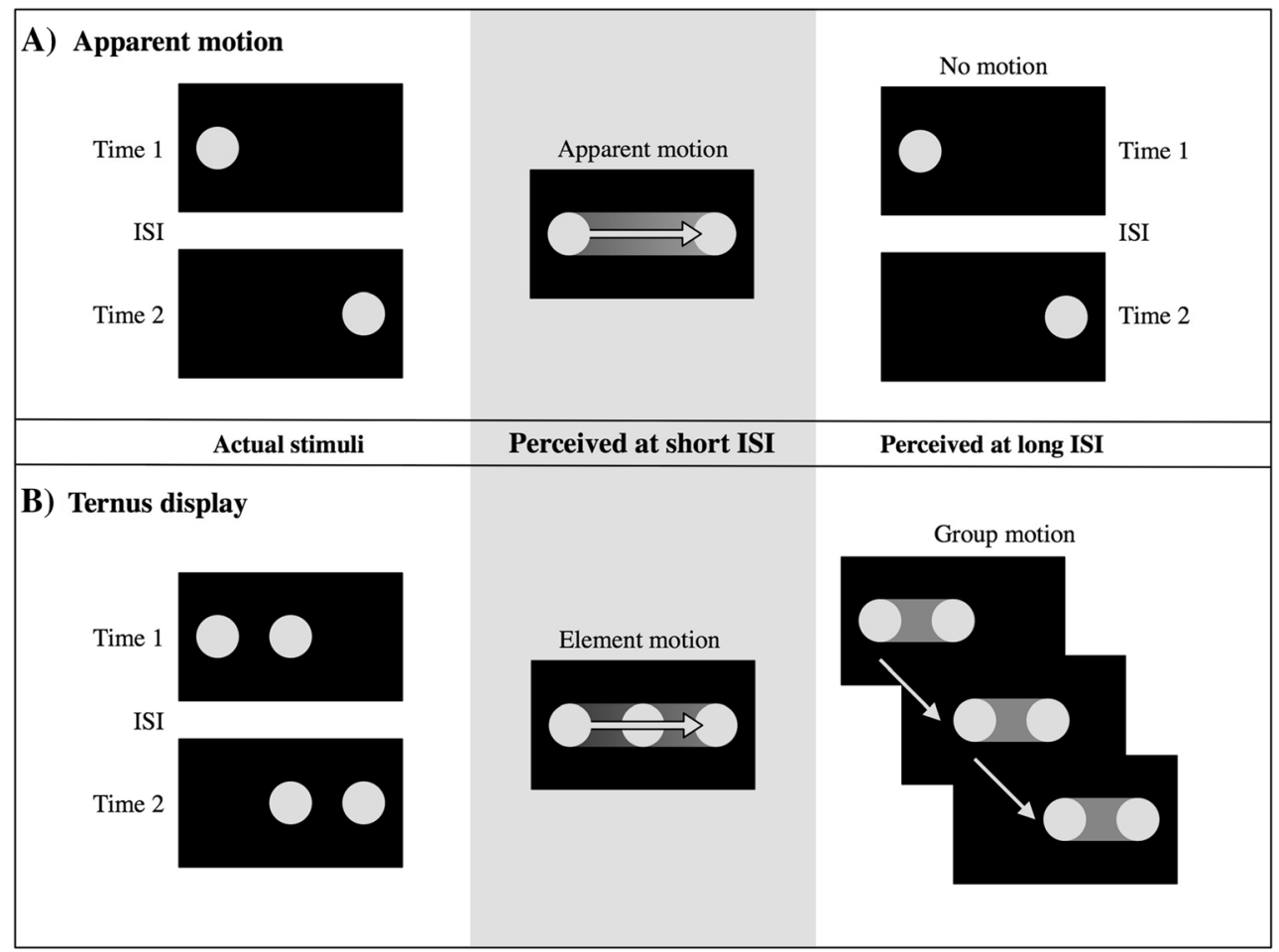

Fig. 1 The two visual illusions used in the study. (A) Experiment 1: Apparent motion. When two spatially separated, successive visual stimuli are presented with a short enough interstimulus interval (ISI), people observe apparent motion, whereby the first stimulus appears to move to the location of the second stimulus. When the ISI is too long, people see two separate and successive stimuli. (B) Experiment 2: Ternus display. Two successive, horizontally displaced pairs of visual stimuli are presented such that the rightmost stimulus in the first pair is located in the same position as the leftmost stimulus in the second pair. With a short enough ISI, people see element motion, whereby the outermost stimulus leapfrogs over the middle stimulus, which remains stationary. If the ISI is too long, people see group motion, whereby the pair moves in tandem next trials, please remove your hand from the mouse. The display will initiate automatically."

At the beginning of each trial, participants observed a black fixation cross within a small rectangle located in the center of the screen. In the voluntary-action condition, they fixated on the cross until they pressed the mouse button. At that point, a black circle (diameter $=5 \mathrm{~mm}\left[\sim 0.4^{\circ}\right]$, duration $=83 \mathrm{~ms}$ ) immediately flashed on the left side of the rectangle, followed after the designated ISI by an identical circle $15 \mathrm{~mm}$ to the right of the first circle. The midpoint of the apparent motion path was $10 \mathrm{~mm}$ below the fixation point. A total of $600 \mathrm{~ms}$ after the second circle had disappeared, the fixation cross disappeared as well, and two lines of text appeared below the rectangle, reminding the participants to press " 1 " if they saw motion or " 2 " if they did not. The chosen response was highlighted for $500 \mathrm{~ms}$, and another trial began after a 500$\mathrm{ms}$ intertrial interval. The experiment was programmed with the OpenSesame software package (Mathôt, Schreij, \& Theeuwes, 2012). A schematic of the apparent-motion stimuli is shown in Fig. 1A.

The no-action condition was the same as the voluntaryaction condition, except that instead of pressing the mouse button, participants fixated on the cross until the first circle automatically appeared after a random delay (from a uniform distribution of 500-3,500 ms). The onset of the first circle was paired with a recorded mouse click sound so as to equate the auditory stimulation across conditions (see Humphreys \& Buehner, 2010; Kawabe, Roseboom, \& Nishida, 2013). We did not include a "passive" movement condition in which the finger was moved by something other than the participant, because we were interested in comparing the effects of voluntary actions to passive viewing. We were concerned that having one's finger moved by a machine - as is usual when this condition is included - might startle, surprise, or lead to reorienting attention away from the main task, which might interfere with perception. Each participant completed the task in approximately $30 \mathrm{~min}$.

Interval estimation task The interval estimation task was conducted after participants had completed the apparentmotion task. It featured a 2 (Action Condition: voluntary action, no action) $\times 4$ (ISI: $50,150,250,350 \mathrm{~ms}$ ) withinparticipants design. Action condition was manipulated within four counterbalanced blocks of 40 trials each, and ISI was randomized between the trials in each block, resulting in 20 trials of each ISI-action condition pair and a total of 160 trials per participant. After each display, participants responded to the question "How long was the interval between the two 
circles, in milliseconds?" by typing a number with the computer keyboard. This procedure followed that of Engbert et al. (2007). Participants were informed at the beginning of the task that the interval would always be between 1 and $500 \mathrm{~ms}$. The task took approximately $30 \mathrm{~min}$.

\section{Results}

Apparent motion Although we chose the range of ISIs to capture the ISIs over which most participants see apparent motion as a sharply declining function of ISI (Kolers \& Pomerantz, 1971), there is variability in this percept (Ekroll, Faul, \& Golz, 2008). For our purposes, there would be no possibility of measuring a difference between conditions if a participant failed to show any sensitivity to ISI in the range of ISIs investigated. We therefore rejected participants who did not show at least a $10 \%$ decline in apparent-motion responses from the shortest ISI to the longest, averaged over both conditions. This rule excluded four participants and left a final sample size of 20 participants. Additionally, one participant quit the study before the second voluntary-action block. Otherwise, no trials were excluded.

Voluntary actions increased apparent-motion perception, as is shown in Fig. 2. A Bayesian multilevel logistic regression ${ }^{1}$ model showed that voluntary actions increased apparentmotion perception $(\beta$ action $=0.51,95 \%$ CI $[0.07,0.99]$, posterior probability $=98.8 \%, z=2.50, p=.01){ }^{2}$ We estimated the magnitude of the perceptual shift by comparing the average 50\% motion perception thresholds (i.e., the points of subjective equality, PSEs) between conditions. The PSE was $19 \mathrm{~ms}$ higher in the voluntary-action condition, indicating that people perceived apparent motion at a longer ISI in the voluntary-action condition $\left(\mathrm{PSE}_{\mathrm{volun}}=113 \mathrm{~ms}, 95 \% \mathrm{CI}[84\right.$, $142] ; \mathrm{PSE}_{\text {no act }}=95 \mathrm{~ms}, 95 \% \mathrm{CI}[60,126]$; difference in PSEs $($ voluntary action - no action $)=19 \mathrm{~ms}, 95 \%$ CI $[2,38])$. The posterior probability for a positive shift in PSE was 98.3\%.

Interval estimation Before analyzing the interval estimation data, we removed 29 trials - out of a total of 3,040 - because they had responses outside the accepted range (1-500 ms). One

\footnotetext{
${ }^{1}$ We used minimally informative priors to constrain the parameter estimates on the log-odds scale. We used $\operatorname{Normal}(0,100)$ distributions as priors for the population-level (fixed) regression coefficients, and $\operatorname{Cauchy}^{+}(0,4)$ priors for the participant-level (random) coefficient $S D$ s. We also estimated the model using standard maximum-likelihood methods (Bates, Mächler, Bolker, \& Walker, 2015), which led to identical conclusions. For parameters estimated with the Bayesian model, we report the posterior means $(\beta)$ and their associated $95 \%$ credible intervals $(\mathrm{CI}$; the central $95 \%$ of values in the respective marginal posterior distribution). All Bayesian inference was done via Hamiltonian Monte Carlo sampling as implemented in the Stan programming language, and the posterior samples were analyzed using R (Buerkner, 2016; R Development Core Team, 2016; Stan Development Team, 2016).

${ }^{2}$ All $p, t$, and $z$ values are from models estimated with classical maximumlikelihood methods.
}

participant quit the study before the interval estimation task, resulting in 19 participants' data being included in the analysis.

We analyzed the interval estimation data using a Bayesian multilevel linear regression model. As we expected from the previous literature on intentional binding (Engbert et al., 2007), interval estimates were shorter in the voluntary action condition (Fig. 3). On average, participants gave 25-ms shorter estimates in the voluntary-action condition $(95 \%$ CI $[-41,-9.4])[t(18)=$ $-3.47, p=.003]$. ISI and action condition did not interact.

\section{Discussion}

Experiment 1 showed that people saw the illusion of apparent motion at longer ISIs when they had voluntarily initiated the display, as compared to when they had viewed it passively. This differential perception of the visual illusion of motion appears to reflect voluntary-action-induced compression of subjective time.

A possible concern, though, is that voluntary actions might somehow bias or prime participants to report motion. The fact that the finger moved might have triggered the idea of movement, which was then reported as more apparent movement in that condition. In contrast to this concern, the perception of apparent motion is usually thought to be cognitively impenetrable (Dawson, 1991), making it unlikely that some selective priming of "motion" could have taken place in the voluntaryaction condition. Nevertheless, to address the possible issue of priming or biasing, in Experiment 2 we used the Ternus display, in which motion is perceived at both short and long ISIs, but different types of motion as the ISI lengthens. Using another illusion also allowed us to conceptually replicate our findings from Experiment 1.

\section{Experiment 2a}

\section{Method}

Experiment 2a was similar to Experiment 1, except that the Ternus display was used (Fig. 1B). The Ternus display consists of two horizontally aligned pairs of visual stimuli. The first pair is shown such that the rightmost object is at the center of the display; then, after a brief ISI, another pair of stimuli is presented, such that now the leftmost object is at the center of the display. If the ISI is short, people see element motion, whereby the outermost object jumps across the display and the central object seems to remain stationary. At long ISIs, people see group motion, whereby the pair of objects seems to move together.

Participants Thirty-six Columbia University undergraduate students participated in the experiment in exchange for course credit. The increase in sample size allowed us to repeat each 
A)

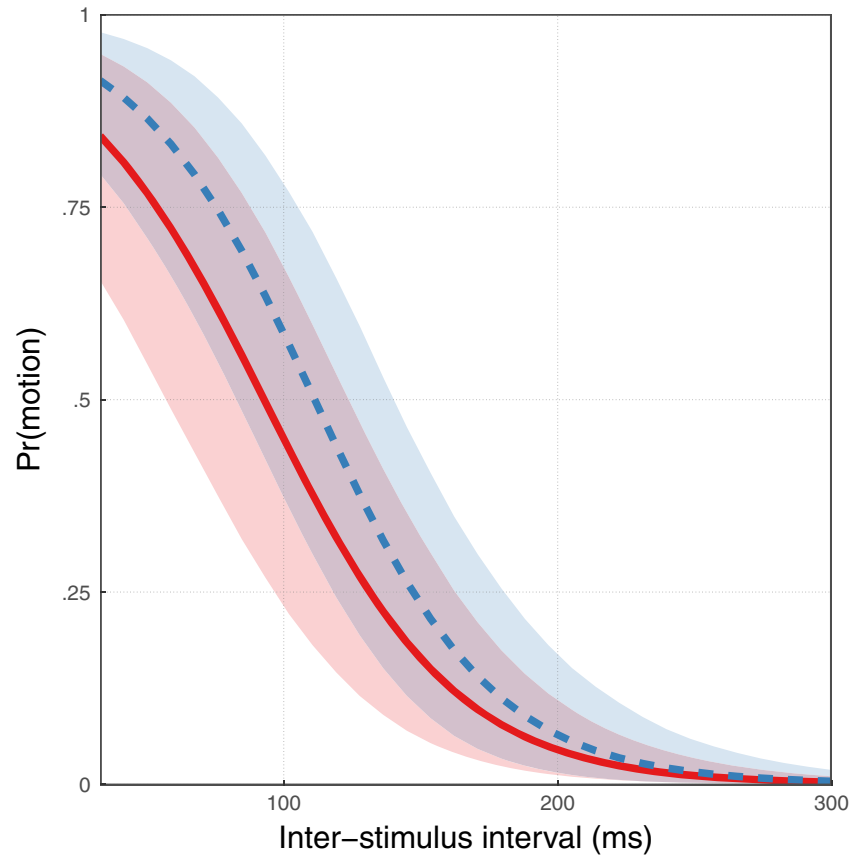

B)

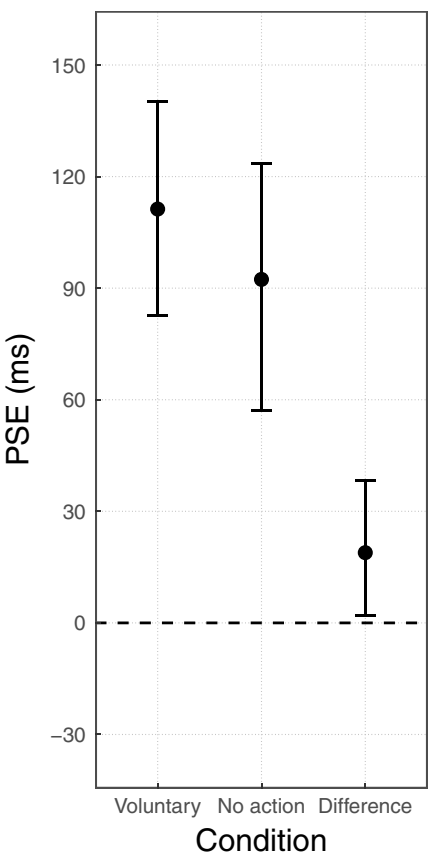

Fig. 2 Multilevel logistic regression model of the apparent motion task, Experiment 1. (A) Average model-predicted probabilities of apparent motion for the no-action (solid line) and voluntary-action (dashed line) conditions, with $95 \%$ credible intervals (CIs) as lighter shades of the different line colors. (B) Points of subjective equality (PSEs) in both experimental conditions, as well as their difference (voluntary action no action). Error bars indicate $95 \%$ CIs
A)

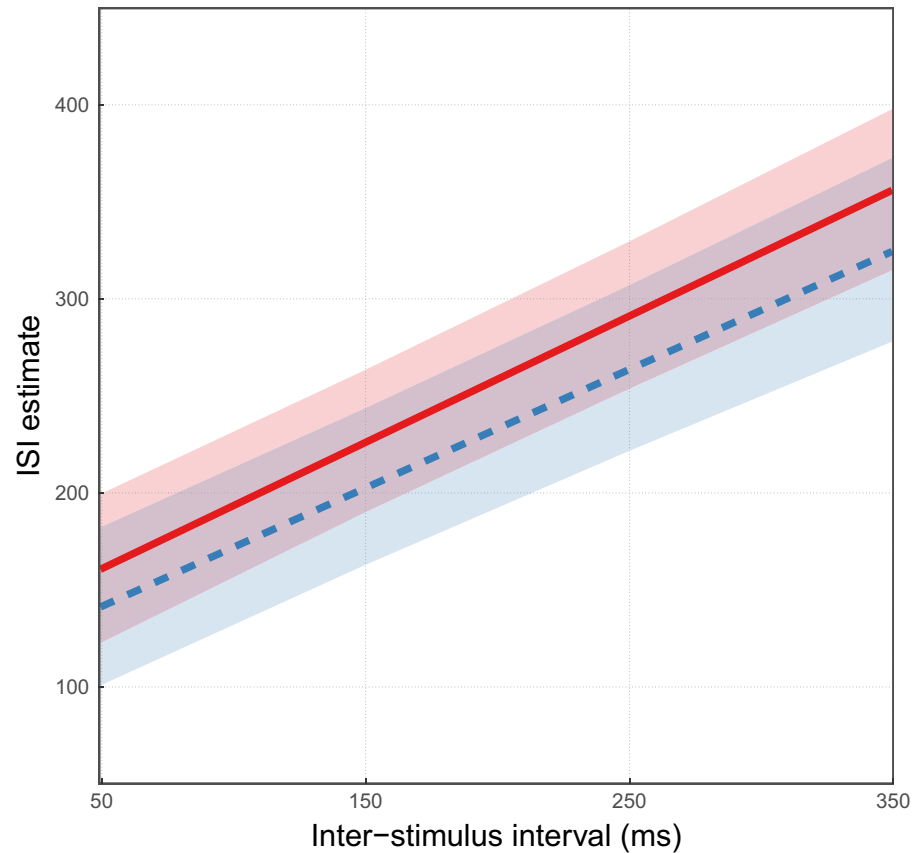

B)

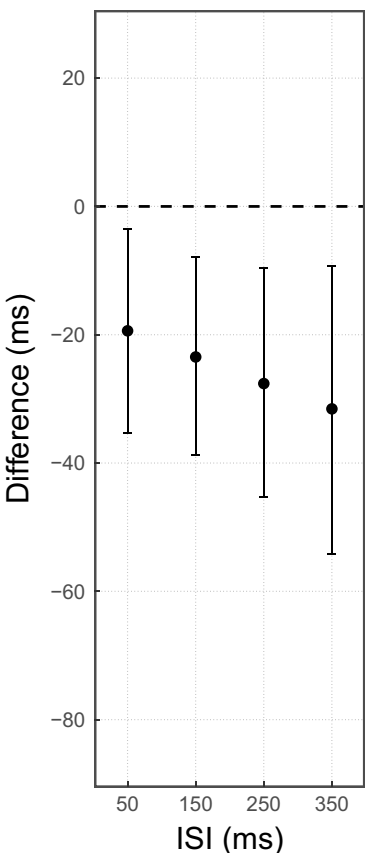

Fig. 3 Multilevel regression model of the interval estimation task in Experiment 1. (A) Average model-predicted interval estimates for the no-action (solid line) and voluntary-action (dashed line) conditions, with $95 \%$ credible intervals (CIs) as lighter shades of the different line colors. (B) Difference in interval estimates (voluntary action - no action) for each ISI; error bars indicate $95 \%$ CIs 
counterbalanced condition six times instead of four times over all participants. The experiment was approved by the Columbia University Internal Review Board and was carried out in accordance with the Psychonomic Society ethical guidelines and with the Declaration of Helsinki.

Ternus display task Here we used a 2 (Action Condition: voluntary action, no action) $\times 10$ (ISI: $0,13,27,40,53,67$, $80,93,107,120 \mathrm{~ms}$ ) within-participants design. The dependent measure - type of motion perceived - was measured as a binary (element or group motion) response. Action condition was manipulated within four counterbalanced blocks of 120 trials each, and ISI was randomized between trials, resulting in 24 trials of each ISI-action condition pair, for a total of 480 trials per participant.

Participants completed the experiment individually, on a Dell desktop computer running at a $75-\mathrm{Hz}$ refresh rate, in a dark testing room. They were seated approximately $60 \mathrm{~cm}$ from the monitor, and wore headphones throughout the study. At the beginning of the study, the participants were instructed that the experiment was about different types of motion percepts. Before the experiment, they read the following instructions:

In this task, we ask you to observe brief visual stimuli on the screen, and report what you see. Specifically, we will show you a display called the Ternus display. In the Ternus display, you will see two circles flash on the screen, then another two circles flash slightly to the right of the first two circles. This can lead to two different types of motion perception. The first, called element motion, looks as if only the outermost circle moved from the left-most position to the right-most position, while the middle circle remained stationary. The second type of motion perception is called group motion, and looks as if both circles moved right.

They also saw an image similar to Fig. 1B. They then passively observed 34 demonstration trials of the Ternus display; 12 trials with short intervals ( 0 - and 13-ms ISIs), 12 trials with long intervals (120- and 133-ms ISIs), and 10 trials with the intervals used in the actual experiment. Participants did not provide responses during the demonstration trials.

The experiment was identical to Experiment 1, apart from the following changes to the visual stimuli. After the fixation cross disappeared, two dark gray Gaussian circles (diameter $=$ $8 \mathrm{~mm}\left[\sim 0.7^{\circ}\right]$, frequency $=0.001, S D=6$, duration $=67 \mathrm{~ms}$ ) flashed under the fixation cross, one directly under it, and the other one displaced $16 \mathrm{~mm}$ to the left, followed after the designated ISI by two identical circles, one again in the middle, and the other one displaced $16 \mathrm{~mm}$ to the right. The middle circle was always placed $8 \mathrm{~mm}$ below the fixation point. A total of $600 \mathrm{~ms}$ after the second pair of circles had disappeared, the fixation cross also disappeared, and two lines of text ("Element motion"; "Group motion") appeared below the rectangle. Participants provided unspeeded responses, and by pressing "E" for element motion or "G" for group motion. The chosen response was highlighted for $500 \mathrm{~ms}$, and another trial began after a $500-\mathrm{ms}$ intertrial interval. Each participant completed the task in approximately $30 \mathrm{~min}$.

\section{Results}

We used the same exclusion criteria as in Experiment 1: Three participants were rejected because their element motion responses were not sensitive to changes in the ISI. Additionally, two participants reversed the response buttons; their data were included after reversing their responses.

People persisted in seeing the illusory percept associated with shorter ISIs (element motion) at longer ISIs when they had initiated the display with voluntary action (Fig. 4). A Bayesian multilevel logistic regression model (see note 1 above) showed that voluntary action increased element motion perception $(\beta$ action $=0.38,95 \%$ CI $[0.17,0.60]$, posterior probability $=99.9 \%, z=3.81, p=.0001)$. We quantified the perceptual shift using the $50 \%$ element motion thresholds (PSEs). The PSE was $7.7 \mathrm{~ms}$ higher in the voluntary-action condition than in the passive condition $\left(\mathrm{PSE}_{\mathrm{volun}}=49 \mathrm{~ms}\right.$, 95\% CI $[43,56] ; \mathrm{PSE}_{\text {no act }}=41 \mathrm{~ms}, 95 \%$ CI $[35,48]$; difference between PSEs (voluntary - no action) $=7.7 \mathrm{~ms}, 95 \% \mathrm{CI}$ $[3.6,11])$. The posterior probability for a positive difference in PSEs was very high $(99.95 \%)$.

\section{Discussion}

Experiment 2a showed that people were more prone to observe a type of motion (element motion) associated with short ISIs when they had voluntarily initiated the display. These results discount the idea that voluntary actions biased or primed participants to report motion, because both possible percepts in the Ternus display are types of motion. Instead, voluntary actions selectively increased the perception of a type of motion associated with short ISIs. However, due to a computer error, the counterbalances were not appropriately rotated across participants. To correct this error, and to replicate our findings, we fixed the counterbalance rotation and conducted a direct replication of Experiment 2a.

\section{Experiment 2b}

\section{Method}

Experiment $2 \mathrm{~b}$ was a direct replication of Experiment $2 \mathrm{a}$, with the computer error in counterbalancing corrected and using different participants. 

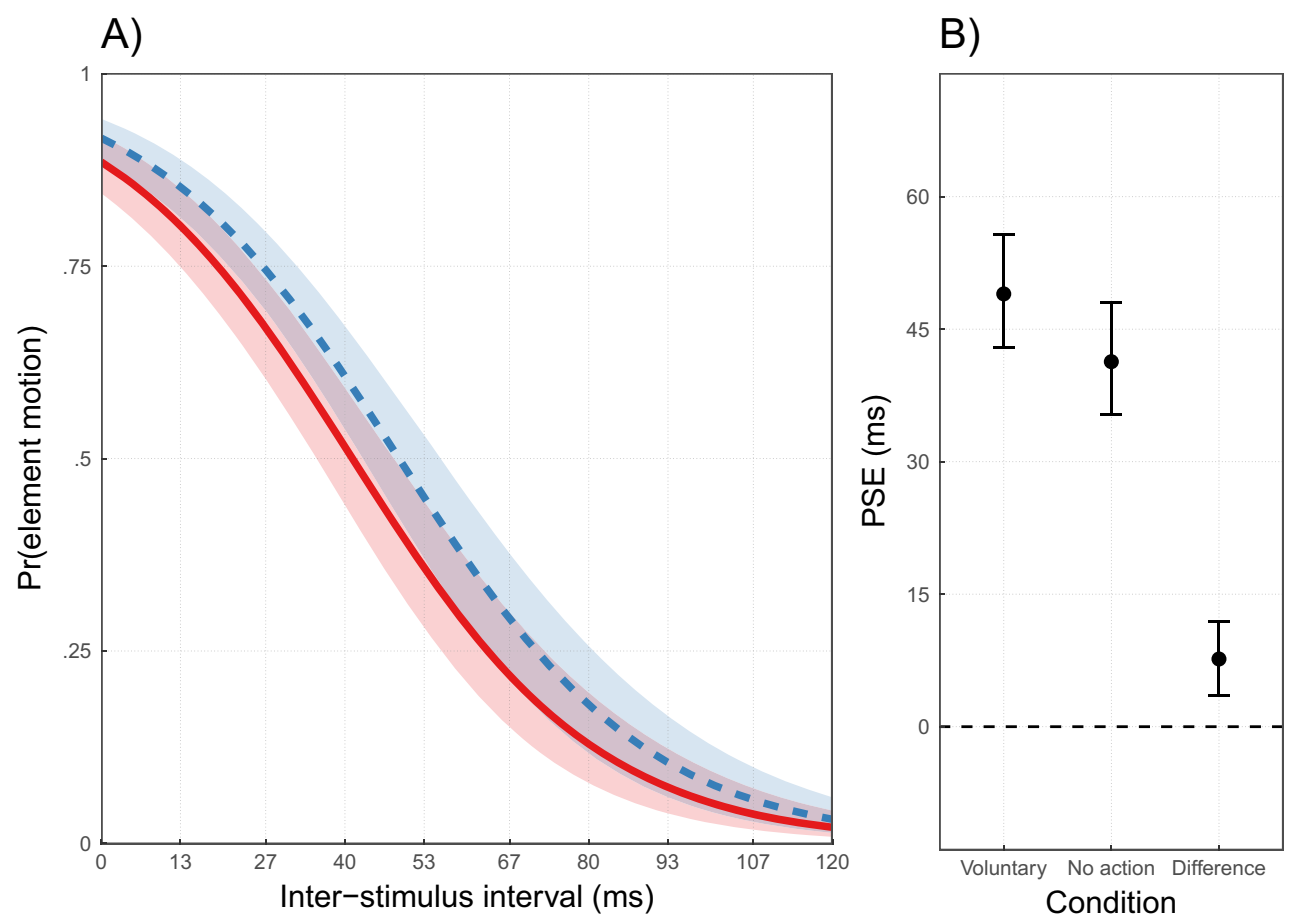

Fig. 4 Multilevel logistic regression model of the Ternus display task in Experiment 2a. (A) Average model-predicted probabilities of element motion responses for the no-action (solid line) and voluntary-action (dashed line) conditions, with $95 \%$ credible intervals (CIs) as lighter

shades of the different line colors. (B) Points of subjective equality in both experimental conditions, and their difference (voluntary action - no action; error bars indicate $95 \%$ CIs)

Participants Thirty-eight Columbia University undergraduate students participated in the experiment in exchange for course credit. The experiment was approved by the Columbia University Internal Review Board and was carried out in accordance with the Psychonomic Society ethical guidelines and with the Declaration of Helsinki.

\section{Results}

We used the same exclusion criteria as in Experiment 1: One participant was rejected because of element motion responses that were not sensitive to changes in the ISI. One participant reversed the response buttons, and these data were included after reversing the responses.

The results replicated those of Experiment 2a: A Bayesian multilevel logistic regression model (see note 1 above) showed that voluntary action increased element motion perception $(\beta$ action $=0.25,95 \% \mathrm{CI}[0.002,0.503]$, posterior probability $=$ $97.6 \%, z=2.17, p=.03$ ). We quantified the perceptual shift using the $50 \%$ element motion thresholds (PSEs). The PSE was $6.3 \mathrm{~ms}$ higher in the voluntary-action condition than in the passive condition $\left(\mathrm{PSE}_{\mathrm{volun}}=50 \mathrm{~ms}, 95 \% \mathrm{CI}[44,57]\right.$; $\mathrm{PSE}_{\mathrm{no}}$ ${ }_{\text {act }}=44 \mathrm{~ms}, 95 \% \mathrm{CI}[35,53]$; difference between PSEs (voluntary - no action $)=6.3 \mathrm{~ms}, 95 \% \mathrm{CI}[0.13,12.7])$. The posterior probability for a positive difference in PSEs was high $(97.72 \%)$. These results are illustrated in Fig. 5.

\section{Discussion}

Experiments $2 \mathrm{a}$ and $2 \mathrm{~b}$ showed that people observe element motion at longer ISIs following voluntary action than following passive observation. These results support the hypothesis that voluntary action leads to a perceptual compression of time.

Even so, there remained a possible alternative explanation for the results that implicated differences in people's attention in the two conditions. It is possible that people perceived the stimuli differently between the two conditions not because time was subjectively compressed, but rather because voluntary actions allow for better attention, preparation, or ability to predict the timing of the stimuli. For example, previous studies suggested that voluntary actions can have effects on perception because they allow for the accurate prediction of when or what is about to happen (Waszak, Cardoso-Leite, \& Hughes, 2012). We thought that attention allocation and differential predictability of the stimuli were unlikely explanations for the present results. First, increased attention should make the stimuli more distinct. Furthermore, it has sometimes been found that increased attention to a task lengthens, rather than shortens, perceived duration (Macar, Grondin, \& Casini, 1994). If that were the case, and if participants 

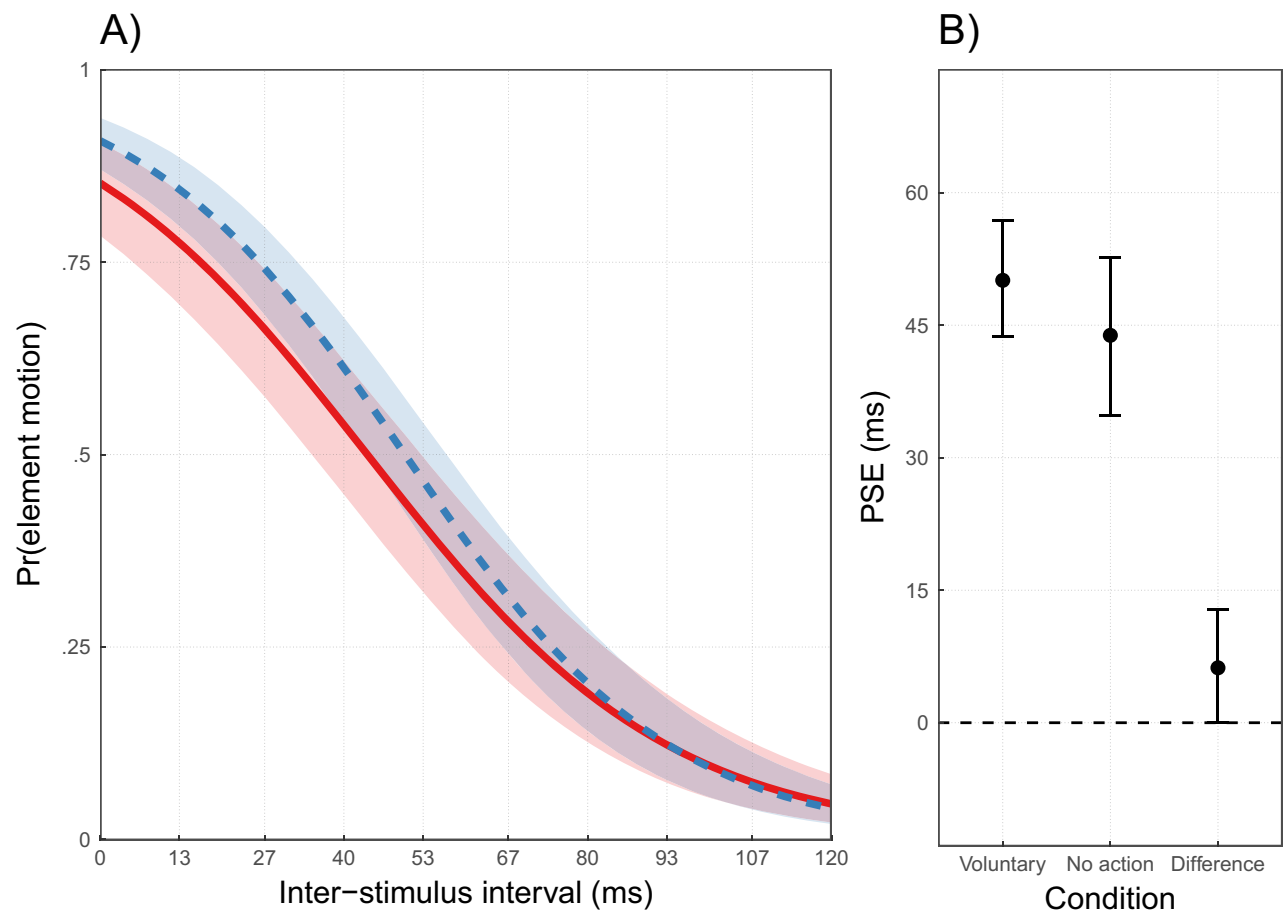

Fig. 5 Multilevel logistic regression model of the Ternus display task in Experiment 2b. (A) Average model-predicted probabilities of element motion responses for the no-action (solid line) and voluntary-action (dashed line) conditions, with $95 \%$ credible intervals (CIs) as lighter

shades of the different line colors. (B) Points of subjective equality in both experimental conditions, and their difference (voluntary action - no action; error bars indicate $95 \%$ CIs)

were paying more attention in the voluntary-action condition, they should have seen less rather than more apparent motion: The apparent time intervals should have been expanded, not compressed. For these reasons, we thought that an attention allocation explanation of our results was unlikely. Nevertheless, increased attention to, preparation for, or predictability of the visual stimuli in the voluntary action condition did seem plausible, and the effects of attention in this paradigm were unknown.

To evaluate the differential-attention explanation of our results, we conducted a final control experiment in which we manipulated participants' ability to prepare, attend, and predict the stimuli. We employed the manipulations used in classic preparation experiments (e.g., Behar \& Adams, 1966), using warning signals to alert participants to an upcoming stimulus, as the basis of our design. In this final experiment, participants observed the Ternus display in two conditions: (1) In the warning signal condition, they heard a tone $1 \mathrm{~s}$ before the onset of the first visual stimulus, and (2) in the no-warning signal condition, they were not forewarned about the onset of the visual stimuli. According to the attentional view of our effects, the first (warning) condition should be similar to the voluntary-action condition, and the second (no-warning) condition should be like the passive condition. If attention or predictability mediates the effects of action, people should perceive element motion at longer ISIs in the warning condition than in the no-warning condition.

\section{Experiment 3}

\section{Method}

The stimuli and task were identical to those of Experiment 2, except that the voluntary-action manipulation was replaced with a warning signal manipulation. In the warning signal condition, a tone $(600-\mathrm{Hz}$ sine wave, duration $100 \mathrm{~ms}$, clearly audible but not painful volume) was played through the headphones $1 \mathrm{~s}$ prior to the initiation of the Ternus display. We chose a 1-s interval between the warning signal and the first visual stimulus ("foreperiod") on the basis of the previous literature (Behar \& Adams, 1966; Langner, Steinborn, Chatterjee, Sturm, \& Willmes, 2010), while attempting to minimize cross-modal integration between the warning tone and visual stimulus that could be caused by too short a foreperiod (e.g., Fendrich \& Corballis, 2001). In the nowarning condition, the Ternus display started after a random delay as in Experiment 2, without any tone.

Participants Thirty-six Columbia University undergraduate students participated in the experiment in exchange for course credit. The experiment was approved by the Columbia University Internal Review Board and was carried out in accordance with the Psychonomic Society ethical guidelines and with the Declaration of Helsinki. 


\section{Results}

We used the same exclusion criteria as in Experiment 1: Three participants were rejected because their element motion responses were not sensitive to changes in the ISI. One participant reversed the response buttons, and these data were included after reversing the responses.

The results (see note 1 ) showed that the warning signal had no effect on perception of the Ternus display $(\beta$ warning $=-$ $0.03,95 \%$ CI $[-0.20,0.15]$, posterior probability $=38.4 \%, z=$ $-0.36, p=.72$ ). The PSE was $0.5 \mathrm{~ms}$ shorter in the warning signal condition than in the no-warning condition $\left(\mathrm{PSE}_{\mathrm{warn}}=\right.$ $50 \mathrm{~ms}, 95 \%$ CI $[43,58]$; $\mathrm{PSE}_{\text {no warn }}=51 \mathrm{~ms}, 95 \%$ CI $[43,59]$; difference between PSEs (warning - no warning) $=-0.5 \mathrm{~ms}$, $95 \%$ CI $[-3.9,3.0])$. The posterior probability for a positive difference in PSEs was very low $(38.3 \%)$. These results are illustrated in Fig. 6.

We then asked whether the action and warning signal effects were statistically different from each other, by estimating the same model as above, but using all three Ternus experiments' data and including Condition $\times$ Experiment and ISI $\times$ Experiment interaction terms. This analysis confirmed that the effect of voluntary action was not different across Experiments $2 \mathrm{a}$ and $2 \mathrm{~b}(\beta=-0.08,95 \%$ CI $[-0.36,0.21])$ and that the effect of the warning signal was smaller in Experiment 3 than was the effect of voluntary action in Experiments $2 \mathrm{a}(\beta=-0.38,95 \%$ CI $[-0.67,-0.08])$ and $2 \mathrm{~b}$ $(\beta=-0.30,95 \%$ CI $[-0.59,-0.01])$. In summary, these results showed that the warning signal had no effect on participants' perception of the visual illusions, reinforcing the idea that voluntary actions modulated perception of the illusions through changes in time perception, instead of changes in attention, preparation, or the predictability of the stimuli.

\section{General discussion}

Experiments 1, 2a, and 2b showed that voluntary action produced changes in people's perceptions of visual motion. In all three cases, illusions of motion persisted at longer ISIs following voluntary action than during passive viewing, suggesting that voluntary action compresses subjective time. Although attentional factors can sometimes influence time perception (Ivry \& Schlerf, 2008; Zakay \& Block, 1996), and it seemed plausible that participants might have paid more attention to the task in the voluntary-action condition, Experiment 3 showed that helping people attend to the task with an auditory warning signal had no impact on the perception of the visual illusions. Experiment 3 also ruled out another possible explanation for the observed effects: It is possible that actions modulate how their effects are perceived by means of predictability; that is, actions may allow robust prediction of what is about to happen and when (e.g., Waszak et al., 2012), and therefore the actions may not have been important, but only
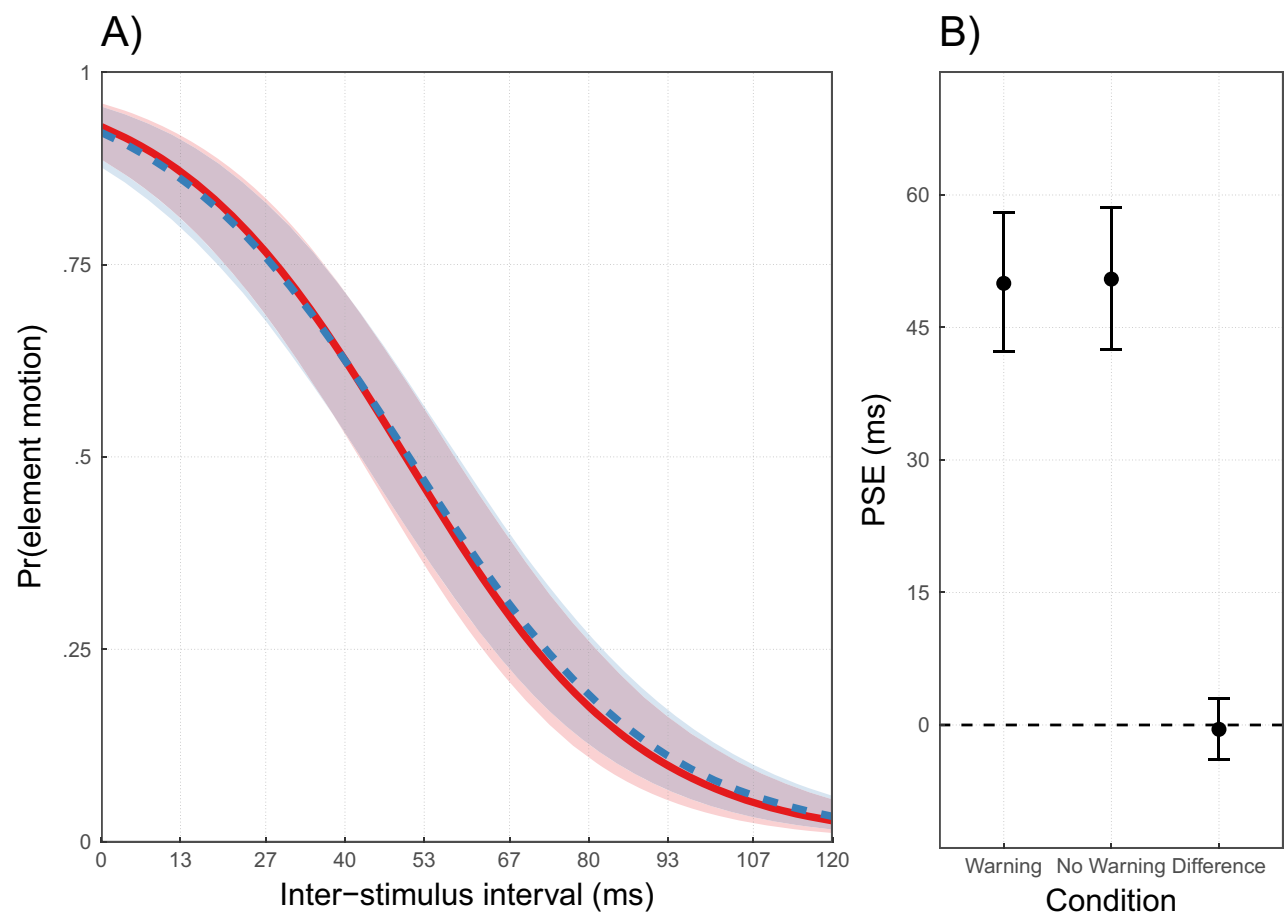

Fig. 6 Multilevel logistic regression model of the Ternus display task in Experiment 3. (A) Average model-predicted probabilities of element motion responses for the no-warning (solid line) and warning sound (dashed line) conditions, with $95 \%$ credible intervals (CIs) as lighter

shades of the different line colors. (B) Points of subjective equality in both experimental conditions, and their difference (warning - no warning; error bars indicate $95 \% \mathrm{CIs}$ ) 
that the stimuli were predictable. In Experiment 3, a warning sound made the stimuli predictable (in comparison to the nowarning condition), yet had no effect on how the illusions were perceived, showing that the differential perception of visual motion was not due to changes in stimulus predictability alone.

What, then, might have caused action to change how the visual illusions were seen? The main idea that has been forwarded, and that is consistent with our data, is that voluntary action compresses subjective time. We will return to this explanation shortly. However, before doing so, another possibility deserves consideration: It is possible that voluntarily initiated actions preactivate the perceptual representations of their effects (Hommel, Müsseler, Aschersleben, \& Prinz, 2001; Waszak et al., 2012). Participants in the present experiments may have learned to anticipate the second stimulus (Time 2 in Fig. 1) when they initiated the display with a voluntary button press, leading to a preactivation or priming of the stimulus at Time 2 . If this occurred, it is conceivable that the stimulus at Time 2 reached the threshold of conscious awareness faster in the voluntary than in the passive condition, and that this priming is what made the second stimulus seem closer in time to the initial one.

Although this explanation is possible, it does not fit easily with the findings of Experiment 3. If anticipation alone is sufficient to preactivate the sensory representation of upcoming events, the warning signal should also have increased the perception of element motion. But Experiment 3 did not show such an effect. However, perhaps voluntary action entails thinking about, and hence priming, the representation of the effect, whereas external warning signals that reduce temporal uncertainty (Waszak et al., 2012) do not prime the effect. Thus, the voluntary-action-related priming account remains a possible explanation of our results. Furthermore, if this account is correct - that voluntary actions preactivate the perceptual representation of their sensory consequences, thus leading them to reach conscious awareness earlier-our findings would have strong implications for current debates about the possibility of the cognitive penetrability of visual perception (e.g., Firestone \& Scholl, 2016), because in the present experiments actions did alter participants' perceptual experiences. More research will be needed to investigate this exciting possibility.

Finally, we think that the most plausible explanation of the present results is that voluntary action directly altered time perception, resulting in a change in perception that was neither purely retrospective in nature, nor due to either attention or stimulus predictability. Although some have suggested that the intentional-binding effect reflects shifts in event timing in relation to one another, rather than a modulation of subjective time itself (Eagleman, 2008; Stetson et al., 2006), it seems more parsimonious to posit that an internal clock mechanism is directly affected. According to this explanation, voluntary actions cause a temporal-rate shift in an internal clock. Internal-clock models postulate that an internal clock tracks time by generating evenly spaced ticks, wherein the number of ticks indicates the magnitude of time passed (see, e.g., Gibbon, Church, \& Meck, 1984; Wearden, 2008). Voluntary actions could therefore temporarily slow down this internal clock, giving rise to fewer ticks during an interval and resulting in both shortened judged time and, more importantly, altered visual illusions of motion.

Author note M.V. and J.M. designed the study. M.V. programmed the experiment, collected and analyzed the data, and drafted the manuscript. M.V. and J.M. wrote and approved the final version of the manuscript for submission. We thank Paul Bloom, Lauren Burns-Coady, Michael Helzer, Kelsey McLeod, Peter Balsam, Cathleen Moore, and the James S. McDonnell Foundation (Grant No. 220020166). This work, in part, contributed to the first author winning the Best Student Poster award (2015 Association for the Scientific Study of Consciousness Annual Meeting) and the Edward E. Smith Memorial Award in Cognitive Neuroscience.

\section{References}

Bates, D., Mächler, M., Bolker, B., \& Walker, S. (2015). Fitting linear mixed-effects models using lme4. Journal of Statistical Software, 67, 1-48. doi:10.18637/jss.v067.i01

Behar, I., \& Adams, C. K. (1966). Some properties of the reaction-time ready-signal. American Journal of Psychology, 79, 419-426. doi:10. 2307/1420882

Buerkner, P.-C. (2016). brms: Bayesian regression models using Stan. Retrieved from http://CRAN.R-project.org/package=brms

Dawson, M. R. (1991). The how and why of what went where in apparent motion: Modeling solutions to the motion correspondence problem. Psychological Review, 98, 569-603. doi:10.1037/0033-295X.98.4.569

Eagleman, D. M. (2008). Human time perception and its illusions. Current Opinion in Neurobiology, 18, 131-136. doi:10.1016/j.conb.2008.06.002

Ekroll, V., Faul, F., \& Golz, J. (2008). Classification of apparent motion percepts based on temporal factors. Journal of Vision, 8(4), 31. doi: $10.1167 / 8.4 .31$

Engbert, K., Wohlschläger, A., Thomas, R., \& Haggard, P. (2007). Agency, subjective time, and other minds. Journal of Experimental Psychology: Human Perception and Performance, 33, 1261-1268. doi:10.1037/0096-1523.33.6.1261

Fendrich, R., \& Corballis, P. M. (2001). The temporal cross-capture of audition and vision. Perception \& Psychophysics, 63, 719-725. doi: 10.3758/BF03194432

Firestone, C., \& Scholl, B. J. (2016). Cognition does not affect perception: Evaluating the evidence for 'topdown' effects. Behavioral and Brain Sciences, 39. doi:10.1017/S0140525X15000965

Gibbon, J., Church, R. M., \& Meck, W. H. (1984). Scalar timing in memory. Annals of the New York Academy of Sciences, 423, 5277. doi:10.1111/j.1749-6632.1984.tb23417.x

Haggard, P., Clark, S., \& Kalogeras, J. (2002). Voluntary action and conscious awareness. Nature Neuroscience, 5, 382-385. doi:10. $1038 / \mathrm{nn} 827$

Hommel, B., Müsseler, J., Aschersleben, G., \& Prinz, W. (2001). The Theory of Event Coding (TEC): A framework for perception and action planning. Behavioral and Brain Sciences, 24, 849-878. doi: 10.1017/S0140525X01000103 
Humphreys, G. R., \& Buehner, M. J. (2010). Temporal binding of action and effect in interval reproduction. Experimental Brain Research, 203, 465-470. doi:10.1007/s00221-010-2199-1

Ivry, R. B., \& Schlerf, J. E. (2008). Dedicated and intrinsic models of time perception. Trends in Cognitive Sciences, 12, 273-280. doi:10.1016/ j.tics.2008.04.002

Kawabe, T., Roseboom, W., \& Nishida, S. (2013). The sense of agency is action-effect causality perception based on cross-modal grouping. Proceedings of the Royal Society B, 280, 20130991. doi:10.1098/ rspb.2013.0991

Kolers, P. A., \& Pomerantz, J. R. (1971). Figural change in apparent motion. Journal of Experimental Psychology, 87, 99.

Langner, R., Steinborn, M. B., Chatterjee, A., Sturm, W., \& Willmes, K. (2010). Mental fatigue and temporal preparation in simple reactiontime performance. Acta Psychologica, 133, 64-72. doi:10.1016/j. actpsy.2009.10.001

Macar, F., Grondin, S., \& Casini, L. (1994). Controlled attention sharing influences time estimation. Memory \& Cognition, 22, 673-686. doi: 10.3758/BF03209252

Mathôt, S., Schreij, D., \& Theeuwes, J. (2012). OpenSesame: An opensource, graphical experiment builder for the social sciences. Behavior Research Methods, 44, 314-324. doi:10.3758/s13428011-0168-7

Moore, J. W., \& Obhi, S. S. (2012). Intentional binding and the sense of agency: A review. Consciousness and Cognition, 21, 546-561. doi: 10.1016/j.concog.2011.12.002

R Development Core Team. (2016). R: A language and environment for statistical computing. Vienna, Austria: R Foundation for Statistical Computing. Retrieved from https://www.R-project.org/
Stan Development Team. (2016). Stan: A C ++ library for probability and sampling (Version 2.14.1). Retrieved from http://mc-stan.org/

Stetson, C., Fiesta, M. P., \& Eagleman, D. M. (2007). Does time really slow down during a frightening event? PLOS ONE, 2, e1295. doi:10. 1371/journal.pone.0001295

Ternus, J. (1926). Experimentelle Untersuchungen über phänomenale Identität. Psychologische Forschung, 7, 81-136. doi:10.1007/ BF02424350

Waszak, F., Cardoso-Leite, P., \& Hughes, G. (2012). Action effect anticipation: Neurophysiological basis and functional consequences. Neuroscience \& Biobehavioral Reviews, 36, 943-959. doi:10. 1016/j.neubiorev.2011.11.004

Wearden, J. H. (2008). Slowing down an internal clock: Implications for accounts of performance on four timing tasks. Quarterly Journal of Experimental Psychology, 61, 263-274. doi:10. 1080/17470210601154610

Wenke, D., \& Haggard, P. (2009). How voluntary actions modulate time perception. Experimental Brain Research, 196, 311-318. doi:10. 1007/s00221-009-1848-8

Wertheimer, M. (1912). Experimentelle Studien über das sehen von Bewegung. Zeitschrift für Psychologie, 61, 161-265.

Williams, S. R., \& Chapman, C. E. (2002). Time course and magnitude of movement-related gating of tactile detection in humans: III. Effect of motor tasks. Journal of Neurophysiology, 88, 1968-1979.

Zakay, D., \& Block, R. A. (1996). The role of attention in time estimation processes. Advances in Psychology, 115, 143-164. 